\title{
Editorial: Improving Pediatric Cardiac Care in India - Expanding Role of Pediatricians
}

\author{
Anita Saxena ${ }^{1}$ \\ Received: 25 June 2015 / Accepted: 2 July 2015 / Published online: 9 November 2015 \\ (C) Dr. K C Chaudhuri Foundation 2015
}

Heart disease in children found its place for the first time in a book on Pediatrics, written by Thomas Morgan Rotch in 1896. This book had seven pages devoted to congenital heart disease. By 1956 the specialty of Pediatric Cardiology had found its place in the Western world. In India, Pediatric Cardiology was practiced as a part of Cardiology specialty in late 1950s. Apart from occasional surgery for patients with congenital heart disease, not much progress was made till late 1960s and early 1970s. Worldwide, technology for pediatric cardiac care advanced rapidly in the last five decades, some of these advances were imported to India. Initially, the availability of good care was limited to very few institutions. As time progressed, the new treatment modalities became available at increasing number of centres all over the country. However, a child with heart disease first comes in contact with a pediatrician. In the current symposia being published in the Indian Journal of Pediatrics, the titles chosen discuss not just the general principles, but also about specific diseases and their management.

Pediatric Cardiology is growing at a rapid pace in India, opening avenues for the latest, state of the art, treatment modalities for children with heart disease. Pediatricians caring for such patients need to be aware of the recent advances for various types of heart diseases, optimal timing of referral, therapeutic options available, protocols for follow up and, short term and

Anita Saxena

anitasaxena@hotmail.com

1 Department of Cardiology, Room \# 29, All India Institute of Medical Sciences, New Delhi 110029, India long term prognosis. The current Pediatric Cardiology symposia aim at equipping the pediatrician to deal with heart diseases in children so as to participate as active stake holders in the management of children with heart disease under their care.

In the first article of this symposium by Dr. S Ramakrishnan, current therapeutic options available for the very serious disease, pulmonary hypertension, are discussed [1]. Pulmonary hypertension has a high morbidity and mortality. The diagnostic work up of children with pulmonary hypertension so as not to miss underlying treatable causes has been well described. He also discusses the challenges faced in India due to presentation of patients in advanced stage of the disease and non availability of some of the effective drugs. Dr Smita Mishra elegantly discusses the cardiac and non cardiac abnormalities in heterotaxy syndromes [2]. Cardiac anatomy as well as extra cardiac associations in such patients and the diagnostic approach is described in detail. Dr P Syamasunder Rao's paper on Fontan operation is highly informative for pediatricians [3]. The various stages of surgery for univentricular hearts are described in detail. Patients with Fontan need lifelong care and the role of pediatrician assumes great significance as prompt diagnosis and treatment of complications are essential for long durability of Fontan circulation. Dr Srinivasan's paper describes a simplified approach to acute management of tachyarrhythmias in children [4]. A number of illustrations make the understanding of arrhythmias much simpler for pediatricians. The final paper is by Dr Parvathy U Iyer. She discusses the various management issues for infants and children with heart disease in intensive care unit [5]. This increasing population in India needs special care in the ICU. Infants and children with acute heart failure, 
severe cyanotic spells, tachy- or bradyarrythmias, cardiac temponade etc. require expert care.

I hope and trust that all these excellent papers published in part II of Pediatric Cardiology Symposium will be of great value to the pediatric community. To excel in the care of children with heart disease, the primary physician including the pediatrician must be able to actively participate as a diagnostician as well as a care taker.

Conflict of Interest None.

Source of Funding None.

\section{References}

1. Ramakrishnan S. Current concepts in management of pulmonary hypertension: fighting the old demon with modern weapons. Indian J Pediatr. 2015. doi:10.1007/s12098-015-1827-y.

2. Mishra S. Cardiac and non-cardiac abnormalities in heterotaxy syndrome. Indian J Pediatr. 2015. doi:10.1007/s12098-015-1925-x.

3. Rao PS. Fontan operation: indications, short and long term outcomes. Indian J Pediatr. 2015. doi:10.1007/s12098-015-1803-6.

4. Srinivasan C. Diagnosis and acute management of tachyarrhythmias in children. Indian J Pediatr. 2015. doi:10.1007/s12098-015-1881-5.

5. Iyer PU. Management issues in intensive care units for infants and children with heart disease. Indian J Pediatr. 2015. doi:10.1007/ s12098-015-1914-0. 